\title{
Noise Reduction of High-Resolution SAR Image over Vegetation and Urban Areas
}

\author{
Vikash Kumar Jha \\ M Tech. Scholar, \\ Department of \\ Electronics \& Communication \\ Engineering, Truba Institute of \\ Engineering and Information \\ Technology Bhopal
}

\author{
Neelesh Gupta \\ H.O.D Department of \\ Electronics \& Communication \\ Engineering, Truba Institute of \\ Engineering and \\ Information Technology Bhopal
}

\author{
Neetu Sharma \\ Asst. prof. Department of \\ Electronics \& Communication \\ Engineering, Truba Institute of \\ Engineering and \\ Information Technology Bhopal
}

\begin{abstract}
Synthetic aperture radar (SAR) has been commonly used in many civilian and military application, and the SAR with moving targets indication is a very hot topic in recent years. As a lot of literatures discussed, if the returns from moving target are method in the same way as the motionless returns, the moving object will appear as an azimuth shift due to range motion and the image of the target will be messy in the azimuth direction due to azimuth motion .The high resolution interferograms above vegetation or urban area are varied, which will break the local stationary theory and make it difficult to get a large number of independent and identically distributed samples for interferometric noise suppression.
\end{abstract}

\section{Keywords}

SAR (synthetic aperture radar), DEMs (digital elevation models) Electromagnetic (EM)

\section{INTRODUCTION}

Interferometric synthetic aperture radar (InSAR) know how to be used to measure the topography or urban subsidence with the phase difference between observations. Interferometric Synthetic aperture radar (InSAR) is one of the most important remote-sensing techniques to obtain topography digital elevation models (DEMs). InSAR illuminate the same ground scene to obtain two or more complex SAR images from slightly different incident angles. Also, the phases of the SAR image pair are process in interferometric way to find out the height of each scattering element in the observed scene (i.e. each pixel of SAR image). This advanced remote-sensing technique, which can work either day or night and through cloudy cover, has many application in the fields of topography, geomorphology, seismology, and so on. As is well known, interferometric phase noise suppression is a key step in InSAR processing procedures. To achieve fine performance of phase noise suppression, it is of extreme importance to obtain more filtering samples that are independent and identically distributed (i.i.d.) from the adjacent pixels. The i.i.d. condition requires that the adjacent pixels must have an identical terrain height. In other words, the height difference of the adjacent pixels inevitably results in poor performance of phase noise suppression, consequently deteriorating the DEM accuracy. Therefore, the effects of neighboring terrain changes must be removed especially for complicated topography, which is usually called topography compensation. Of course, if a prior course DEM of the observed scene is available, the effect of terrain changes on the interferograms can be removed conveniently. Therefore, the interferometric phase filtering is crucial to ensuring the measurement accuracy. Many advanced approaches have been proposed to suppress the interferometric noise. Lee et al used a group of directional windows and an additive noise model to obtain the minimum mean-square estimate of the interferometric phase, called the Lee filter. When the coregistration error is large, $\mathrm{Li}$ et al. takes advantage of the coherence information of neighboring pixels to estimate the interferometric phase. Local fringe frequencies of the interferogram are estimated by the modified multiple-signal classification in and local phase unwrapping in, then the interferogram is filtered.

Synthetic aperture radar (SAR) has already proven its usefulness for the generation of high resolution remote sensing images. Since a SAR employs an active, coherent microwave frequency source of electromagnetic (EM) illumination, it is able to operate under all weather conditions, and without daylight, thus making it an excellent imaging technology for continuous image collection. However, SAR images can suffer from a variety of distortions and artifacts resulting from the image formation process. One such distortion is that of speckle noise which results from the coherent constructive and destructive interference of the backscattered EM radiation from the scattering elements in an area of the ground target equivalent to one resolution cell. As an image artifact, speckle has a granular appearance which corrupts the SAR image information. As noise, speckle may be modeled as a correlated signal dependent random process. It is generally regarded as desirable to reduce or smooth the speckle noise occurring in SAR images. This paper analyzes the heterogeneity of the high-resolution interferometric data over vegetation and urban areas, points out the difficulty in collecting identically distributed (i.i.d.) samples within a local window, and then propose a refined nonlocal filter. In this approach, the similarity distance between the central Pixel and other pixels in the searching window is measured by the normalized probability density function (PDF) of the interferogram. Then, pixels with smaller normalized probability density than the threshold are identified as outliers and removed from the filtering process. Finally, the interferometric phase is estimated by the complex mean filter.

A conventional moving target detection methodology utilizes multi-antennae system, but it generally requires a calibrated and time-invariant radar system, and in practice they are so complex that both high hardware and computation effort are needed in implementation. Recently many gmti methodologies based on a single antenna SAR or a single complex-valued SAR image, such as auto-focusing, antenna beam pattern transforming, and SAR stacks etc. were 
developed and got many effective results. Most of them are based on the moving-target-originated azimuth phase history which is characterized only by two parameters the Doppler shift and the Doppler rate. The former depends on the slantrange velocity component and has been studied deeply before whereas the latter is determined by the azimuth velocity component and is required for correct focusing.

In auto-focusing J. R. Fein up proposed an algorithm to detect along track moving targets in a single SAR imagery by focusing technology used in spotlight SAR. The algorithm divides a complex-valued SAR image into many small patches, and on each patch an auto-focus technique named phase-gradient algorithm (PGA) is utilized to maximize the sharpness of the corrected SAR image. The ratio of the sharpness of the corrected image patch to that of the original patch, referred to as $\mathrm{f} 4$, is adopted as a feature to indicate the presence of a moving target. The computational requirements of the algorithm are dominated by the 1-D FFTs used to transform back and forth between the range-compressed signal history and the image, thus it consumes much time to indicate the targets when the SAR image is in a large scale. Furthermore, the feature value is severely affected by SINR and the selected patch parameters such as the size, background and location. Furthermore, it requires that in each range bin, there is only one moving target and the moving target must exactly be located at center of the patch, otherwise the indicator will give an inaccurate result. D. Weihinget al. constructed a stack of SAR images varying with Doppler frequency modulation rates, and get a series of FM-azimuth slices in each range bin along range direction of the SAR image to indicate moving targets by comparing the changes of the slices.

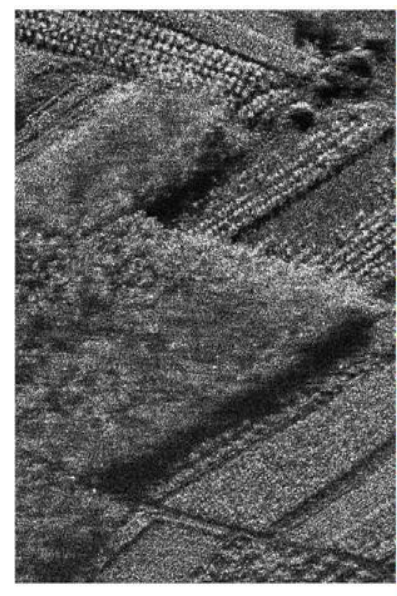

(a) (b)

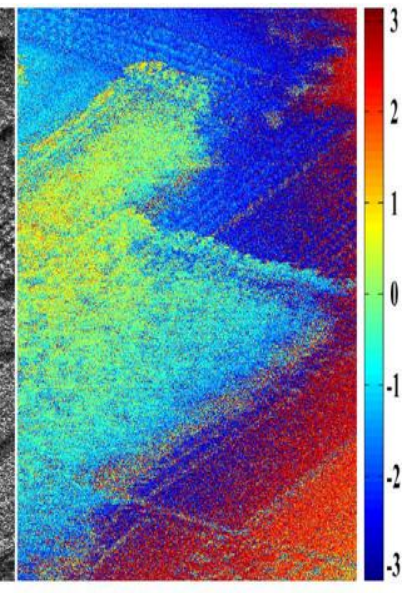

Fig. 1. Ka-band airborne interferometric data of Yantai, China. (a) One-look SAR amplitude image $(966 \times 965$ pixels). (b) Measured interferometric phase.

\section{LITERATURE SURVEY}

All Jin-wei Li et.al.[1] "Noise Filtering of High-Resolution Interferograms Over Vegetation and Urban Areas With a Refined Nonlocal Filter", In proposed work the highresolution interferogram above vegetation area is varied due to open canopy gaps, visible ground, and different plant structure, whereas the interlaced different scattering media are responsible for the heterogeneity of urban areas. The heterogeneity will break the local stationary assumption and degrade the presentation of traditional filters. The developed nonlocal filter proposed in this letter can identify the outliers and remove them from the filtering procedure. The investigational consequences show that the projected method could reduce the interferometric noise effectively and make the edges between different scattering media well preserved. In addition, the reason for the better performance of the pseudo coherence defined as maybe that it can mitigate the effects of the amplitude heterogeneity in the window, and the reason will be investigated further in the future.

Jiao Guo et.al. [2] "Improving the accuracy of local frequency evaluation for interferometric synthetic aperture radar interferogram noise filtering considering large co-registration inaccuracy" In this an inventive technique to estimate the local frequencies of interferogram considering large coregistration error has been presented. Based on the construction of the joint pixel vector and the covariance matrix, the proposed method adopts the separation extent of the signal and noise subspaces as the criteria, optimized to determine the estimates of local frequencies. In the experiments, the simulated data as well as the real ERS-1/2 data has been employed to investigate the performance of the method. The two experiments carried out demonstrate that the proposed method has the ability to acquire accurate estimation of local frequencies even if the co-registration error reaches one pixel. For future InSAR systems with higher spatial resolutions, for example, $1 \mathrm{~m} \times 1 \mathrm{~m}$ or even higher [1,17], it is more difficult to increase the accuracy of image coregistration, so the proposed method would achieve more satisfactory results.

Gaohuan Lv et.al.[3] "Synthetic Aperture Radar Based Ground Moving Target Indicator Using Symmetrical Doppler Rate Matched Filter Pairs", in this proposed work an SDRM filters based GMTI scheme to detect the presence of moving targets and estimate their azimuth velocities in a single complex SAR image. The scheme works fast and effectively during our experiment. A feature criterion to determine the presence of the moving target is defined by the sharpness ratio in a patch in the two derivative SAR images. Simulations showed that the sharpness ratio threshold labeled $\mathrm{ft}$ should satisfy $1: 26 \mathrm{ft} 61: 5$ to indicate a moving target. If the $\mathrm{ft}$ is lower than 1:2, then false alarming happens; if the $\mathrm{ft}$ is larger than 1:5, then false dismissal happens. From our experience, such a condition as $\mathrm{ft}=1: 2$ is a fairly appropriate choice. The algorithm determines the target area automatically so that the moving target will just be located in the center of detected patch, and the background will be excluded as much as it can, therefore the estimate is more accurate when the original SAR image is corrupted by strong noise and strong stationary scatters. The azimuth rapidity estimator is conventional by using an SDRM filter bank. This estimator gives an accurate result when the azimuth velocity is more than $6 \mathrm{~m} / \mathrm{s}$ and the range

Velocity component does not lead to the Doppler ambiguity. However, when the azimuth velocity is with a reduction of than $6 \mathrm{~m} / \mathrm{s}$ or the Doppler uncertainty happens, the AVE may

not come to our expectations. We will try to solve this problem in the future.

Alper Basturk et.al.[4] "Adaptive NEURO-FUZZY Inference System for Speckle Noise Reduction in SAR Images", in this ANFIS maintain method designed for filter of speckle noise in SAR images is presented. The fundamental improvement of the proposed technique over other operators is that it efficiently removes speckle noise while effectively preserve the details and quality in the original image. The superiorities of the projected ANFIS method over further operator in the literature may be summarized as follows It has a extremely 
simple structure. Its primary structure block is a 3-input 1output initial categorize Sugeno type ANFIS. This really simplifies execution. Its presentation does not depend on some parameters that are heuristically determined and externally complete by the client. It is train by means of a very easy simulated image, which can easily be generated in a processor. It uses a 3-by-3 filter window. Some other operators in the literature use filtering windows with superior or with dynamism altering size. It provides an even better performance when repetitively applied to the noisy figure. The boost in presentation varies depending on image property and/or noise density. It is done that the proposed method can be used as a simple but powerful tool for capable removal of speckle noise in SAR images lacking distort valuable information inside the image.

C. Moloney et. al.[5] "Smoothing Speckled Images Using an Adaptive Rational Operator", in this proposed work results of smoothing speckle noise in synthetic aperture radar (SAR) images by means of the multiplicative position estimator, a nearby adaptive form of the homomorphism filter. While the filter does not take account of the SAR speckle noise correlation and encounters some difficulty in obtaining accurate local estimates over small masks, it does perform well in both noise smoothing and edge preservation. Tailoring the filter mask to the image may improve the estimation of the local statistics and hence the overall filtering performance.

\section{METHOD}

In the high-resolution interferogram over vegetation areas, a canopy will possess an oversized range of pixels, and therefore the open cover gaps and visual ground are responsible for the heterogeneous vegetation coverage, which is able to result in the patch-like pattern of interferograms. in addition, plants or trees completely different in height and structure also will cause the heterogeneity of interferograms.previously we have seen that the amplitude backscatter of the urban areas is extremely heterogeneous. For the high-resolution SAR pictures over urban areas, the scattering mechanisms include surface scattering (from playgrounds, streets, public squares, etc.), volume scattering (from forest parks), and point-like scattering (from man-made structures or buildings). the different interlaced scattering media are responsible for the heterogeneity of urban areas. in addition, some pixels aren't even dominated by a single scattering mechanism. Fig. 1 is that the single-pass Ka-band airborne interferometric information over the vegetation area of Yantai, China. both slant range and azimuth resolution is regarding $0.2 \mathrm{~m}$, and therefore the length of baseline is $2.95 \mathrm{~m}$. Fig. 2 is that the single-pass X-band interferometric data of Hainan, China. The resolution is about $0.5 \mathrm{~m}$, and therefore the length of baseline is $5.8 \mathrm{~m}$. Figs. 1(a) and 2(a) show the one-look SAR amplitude images, and pixel grey levels represent amplitudes of backscattering, and Figs. 1(b) and 2(b) are the. From Fig. 1, the plants different in height and structure can be easily seen, and the canopy gaps can be also found in the upper side of the images. Fig. 2 is the epitome of urban areas, and buildings, playground, streets and trees coexist in a limited area. These features cause the heterogeneity of interferograms, which may make the pixels within a local window possess different characteristics and therefore worsen the performance of traditional filters.

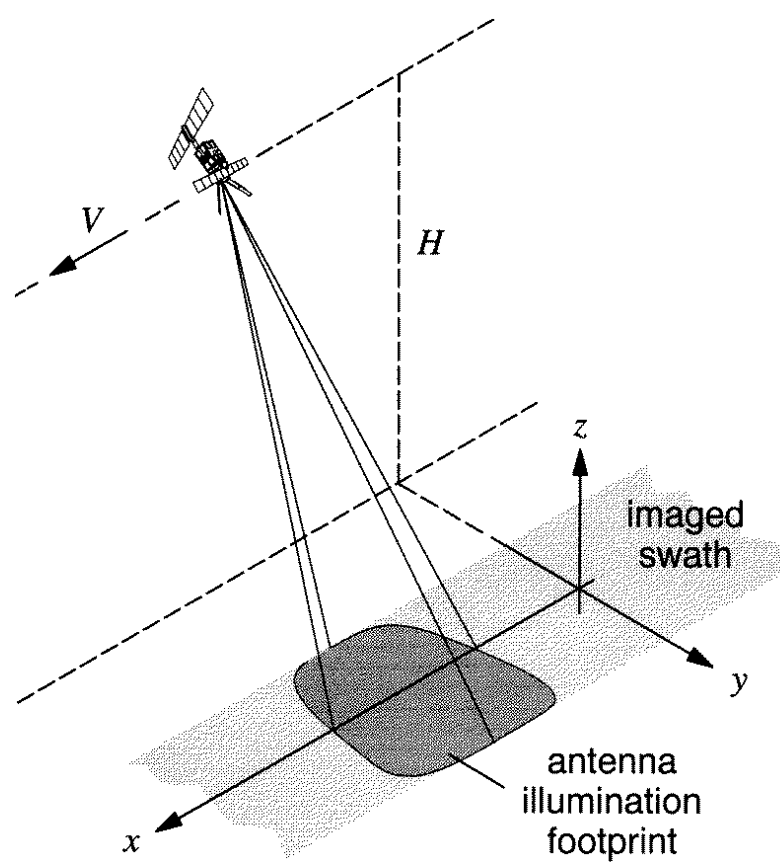

Figure 2. SAR imaging geometry. Frequently used terms are 'along-track' or 'azimuth' for $x$, 'ground range' for $y$, and 'slant range' for the distance of a particular point from the SAR sensor.

$$
\begin{aligned}
\hat{C}_{j s}(i, j)= & \frac{1}{(2 p+1)(2 q+1)} \sum_{u=-p}^{p} \sum_{v=-q}^{q} j s^{\prime}(i+u, j+v) \\
& \times j s^{\prime} H(i+u, j+v)
\end{aligned}
$$

Where $(2 p+1)$ and $(2 q+1)$ are the number of azimuth and range samples of the local window.

Step four: Joint covariance matrix eigen decomposition: The joint covariance matrix $\mathrm{C}^{\wedge} \mathrm{j}_{\mathrm{s}}(\mathrm{i}, \mathrm{j})$ assumed with the dimensions of $\mathrm{M} \times \mathrm{M}$ can be eigen decomposed into from

$$
\hat{c}_{j s}(i, j)=\sum_{k=1}^{K} \lambda_{k} \beta_{s j c}^{(k)} \beta_{s j c}^{(k) H}+\sum_{k=K+1}^{M} \lambda_{k} \beta_{n j c}^{(k)} \beta_{n j c}^{(k) H}
$$

Which, we can obtain M eigenvalues as

$$
\lambda_{1}>\lambda_{2}>\lambda_{3}>\ldots \ldots \ldots . .>\lambda_{k}>>\lambda_{K+1}>\ldots \ldots>\lambda_{M}
$$

The proportion optimization: Maximizing the proportion of $\lambda_{\mathrm{K}}$ to $\lambda_{K+1}$, the corresponding values of $t_{a}$ and $t_{r}$ are the accurate estimation of local frequencies. The estimation of local frequencies can be obtained by performing the five steps above for each pixel pair of the interferogram regardless of the co-registration error, which will be verified in the following section. With the joint covariance matrix Eigen decomposition in the optimal case, the joint subspaces (i.e. the joint noise subspace and the joint signal subspace) can be derived. Finally, the interferometric phase can be determined by projecting the joint signal subspace onto the joint noise subspace.

\section{CONCLUSION}

Synthetic aperture radar (SAR) filtering is a critical giving out step in landscape reconstruction and deformation amount. An SDRM filter match up process is given SAR descriptions and achieves two defocused SAR images that have the same 
defocused environment but dissimilar defocused affecting targets, and then by compare the sharpness of the two images, the moving target are unwavering adaptively and automatically. The high-resolution interferograms over vegetation or urban areas are heterogeneous, which will violate the local stationary assumption and make it difficult to obtain a large number of autonomous and identically distributed samples for interferometric noise suppression.

Given coherence, topography phase, and estimated SAR amount of the pixel to be filtered, the similarity distance between the central pixel and further pixels in the searching window is calculated by the normalize probability density function of the interferogram.

\section{REFERENCES}

[1] Jin-wei Li, Zhen-fang Li, Zheng Bao,Ying-long Hou, and Zhi-yongSuo, "Noise Filtering of High-Resolution InterferogramsOver Vegetation and Urban Areas Witha Refined Nonlocal Filter" IEEE Geos. Remote Sensing Lett., vol. 12, no. 1, pp. 77-81, Jan. 2015.

[2] Jiao Guo, Weitao Zhang, YanyangLiu,Longsheng Fu, "Improving the accuracy of local frequency estimationfor interferometric synthetic aperture radarinterferogram noise filtering considering largecoregistration errors" IET Radar Sonar Navig., vol. 8, iss. 6, pp. 676-684, 2014.

[3] GaohuanLv, Junfeng Wang, and Xingzhao Liu, "Synthetic Aperture Radar Based Ground MovingTarget Indicator Using Symmetrical Doppler RateMatched Filter Pairs",IEEE pp.962-967,2012.

[4] [4]AlperBasturk, M. EminYuksel, "Adaptive NEUROFUZZY Inference System for Speckle NoiseReduction in SAR Images" IEEE 2007.

[5] C. Moloney, G. Ramponi,: 'Smoothing Speckled Images Using an Adaptive Rational Operator', IEEE signal processing letters, vol. 4, no. 3, march 1997
[6] E. Trouvé, M. Caramma, and H. Maître, "Fringe detection in noisycomplex interferograms," Appl. Opt., vol. 35, no. 20, pp. 3799-3806,Jul. 1996.

[7] Monti Guarnieri, A.: 'Using topography statistics to help phaseunwrapping', IET Radar Sonar Navig., 2003, 150, (3), pp. 144-151

[8] Stoica, P., Nehorai, A.: 'MUSIC, maximum likelihood, and Cramer-Rao bound: further results and comparisons', IEEE Trans. Acoust.Speech Signal Process., 1990, 38, (12), pp. 2140-2150

[9] Li, Z.F., Bao, Z., Li, H.: 'Image auto-coregistration and InSARinterferogram estimation using joint subspace projection', IEEE Trans.Geosci. Remote Sens., 2006, 44, (2), pp. 288-297

[10] Li, H., Li, Z.F., Liao, G.S., Bao, Z.: 'An estimation method for InSARinterferometric phase combined with image auto-coregistration', Sci.China, Ser. F, 2006, 49, (3), pp. 386-396

[11] [Liu, N., Zhang, L.R., Liu, X.: 'Multibaseline InSAR height estimationthrough joint covariance matrix fitting', IET Radar Sonar Navig.,2009, 3, (5), pp. 474-483

[12] Wu, N., Feng, D.Z., Li, J.X.: 'A locally adaptive filter of interferometricphase images', IEEE Geosci. Remote Sens. Lett., 2006, 3, (1), pp. 73-77

[13] Lee, J.S., Papathanassiou, K.P., Ainsworth, T.L.: 'A new technique fornoise filtering of SAR interferometric phase images', IEEE Trans.Geosci. Remote Sens., 1998, 36, (5), pp. 1456-1465

[14]Z. F. Li, Z. Bao, H. Li, and G. S. Liao, "Image autocoregistration andInSAR interferogram estimation using joint subspace projection," IEEETrans. Geosci. Remote Sens., vol. 44, no. 2, pp. 288-297, Feb. 2006. 